\title{
Efectividad de un programa de intervención basado en mindfulness para autorregular la atención en niñez de educación primaria
}

\author{
Effectiveness of an Intervention Program Based on Mindfulness to Self-Regulate Care in \\ Primary School Children
}

\section{Efetividade de um programa de intervenção baseado na Mindfulness para auto-regular o cuidado em crianças da escola primária}

Nicol Andrea González-Cortez

Universidad del Bío-Bío

Chillán, Chile

gonzalezcortez.nicol@gmail.com

https://orcid.org/0000-0002-6691-3350

Nelly Lagos-San Martín

Universidad del Bío-Bío

Chillán, Chile

nlagos@ubiobio.cl

https://orcid.org/0000-0002-2029-5219

Recibido • Received • Recebido: 02 / 01 / 2020

Corregido • Revised • Revisado: 30 / 07 / 2021

Aceptado • Accepted • Aprovado: 09 / 10 / 2021

\begin{abstract}
Resumen: La bibliografía afirma que la atención es inherente al aprendizaje, de modo que si esta manifestara alteraciones, la entrada y focalización de las diversas experiencias tendría una amplitud limitada, así como también se afirma que si fuera adecuadamente estimulada, el enriquecimiento de las experiencias sería enormemente fructífero. Debido a la importancia de considerar la atención como un proceso formativo, este estudio se ha planteado el diseño de un programa basado en lineamientos mindfulness para fortalecer la autorregulación de la atención en niñez de educación básica y la evaluación de la efectividad de este mismo en el desarrollo de la atención focalizada, sostenida y dividida. Para ello se contó con una muestra de 46 niños y niñas de tercero año básico de la comuna de Pemuco en Chile, con edades comprendidas entre 8 y 9 años de edad. El enfoque del estudio es de tipo secuencial probatorio, enmarcado en un diseño pre-post test con grupo control. En el grupo control y experimental se midió la variable dependiente, mediante los test CARAS, la subescala CLAVES del test WISC-III y el test CSAT. Los resultados indican un aumento significativo del autocontrol atencional del grupo intervenido respecto del grupo control, avalando el impacto de esta herramienta que se pone a disposición del sistema educativo como un programa efectivo para el fortalecimiento de la autorregulación atencional.
\end{abstract}

Palabras claves: Atención; autorregulación; mindfulness; educación primaria. 
http://doi.org/10.15359/ree.26-1.7

http://www.una.ac.cr/educare

educare@una.ac.cr

\begin{abstract}
The literature states that attention is inherent to learning so that, if attention is altered, the entry and focus of the various experiences would have a limited extent. It is also affirmed that if attention was properly stimulated, the enrichment of the experiences would be enormously fruitful. Due to the importance of considering attention as a formative process, this study has designed a program based on mindfulness guidelines to strengthen the self-regulation of attention in basic education children and the evaluation of its effectiveness in developing focused, sustained, and divided attention. For this purpose, a sample of 46 children from the third grade of elementary school in Pemuco, Chile, aged between 8 and 9 years old, was selected. The focus of the study is of a sequential testing type, framed in a Pre-post test design with a control group. In the control and experimental group, the dependent variable was measured using the CARAS test, the CLAVES subscale of the WISC-III test, and the CSAT test. The results show a significant increase in the self-control of the intervention group compared to the control group, endorsing the impact of this tool that is made available to the education system as an effective program for the strengthening of attentional self-regulation.
\end{abstract}

Keywords: Attention; self-regulation; mindfulness; primary education.

Resumo: A literatura afirma que a atenção é inerente ao aprendizado, de modo que, se manifesta alterações, a entrada e o foco das várias experiências teriam uma extensão limitada. Também é afirmado que, se fosse devidamente estimulado, o enriquecimento das experiências seria enormemente frutuoso. Devido à importância de considerar a atenção como um processo formativo, este estudo considerou o desenho de um programa baseado em diretrizes de mindfulness para fortalecer a autorregulação da atenção em crianças da educação básica e a avaliação de sua eficácia no desenvolvimento de atenção localizada, sustentada e dividida. Para isso, foi incluída uma amostra de 46 crianças da terceira série da comuna de Pemuco, no Chile, com idades entre 8 e 9 anos. $O$ foco do estudo é do tipo sequencial provatório, enquadrado num desenho pré-pós-teste com um grupo controle. No grupo controle e experimental, a variável dependente foi medida usando o teste CARAS, a subescala KEY do teste WISC-III e o teste CSAT. Os resultados indicam um aumento significativo no autocontrole do grupo intervenção comparado ao grupo controle, endossando o impacto dessa ferramenta que é disponibilizada ao sistema educacional como um programa efetivo para o fortalecimento da autorregulação da atenção.

Palavras-chave: Atenção; autorregulação; atenção plena; educação básica.

\title{
Introducción
}

La recepción y procesamiento de información que realiza el estudiando cuando aprende, depende de sus preferencias al seleccionar la información, así como también de la motivación hacia la actividad que demanda su atención. Ya que de ello dependen, entre otras, el tiempo de dedicación a la tarea y el involucramiento en la misma.

Mediante una intervención intencionada, el foco atencional del estudiantado debería aumentar, y paralelamente también los procesos y habilidades cognitivas, tales como la memoria

Nicol Andrea González-Cortez y Nelly Lagos-San Martín

Los artículos de la Revista Electrónica Educare del Centro de Investigación y Docencia en Educación de la Universidad Nacional, Costa Rica, se comparten bajo términos de la Licencia Creative Commons: Reconocimiento, No Comercial, Sin Obra Derivada 3.0 Costa Rica. Las autorizaciones adicionales a las aquí delimitadas se pueden obtener en el correo: educare@una.cr 
http://doi.org/10.15359/ree.26-1.7

y la motivación. Razón por la que se apuesta por generar una propuesta que releve la autonomía perceptiva y el autocontrol de la atención, habilidad transcendental que enmarca la entrada al procesamiento informativo, y con ello, su posterior interpretación, manipulación y uso.

Una de las estrategias que promueve la atención desde una perspectiva interna, optativa y arraigada a la decisión, es Mindfulness, cuyo propósito es considerar la expansión perceptiva de las vivencias, aceptando las experiencias presentes, realzando la conciencia y reduciendo las distracciones, tanto internas como externas. De este modo, el mindfulness impulsa la intencionalidad y genera ejercicios voluntarios para el logro de objetivos atencionales, convirtiéndose en un recurso que posibilita un proceso intrapersonal, en beneficio del desarrollo de la habilidad atencional, situación que genera mayores destrezas y competencias en dicha funcionalidad cognitiva.

\section{Atención y metaatención como procesos de dominio reflexivo}

Los procesos cognitivos desarrollan un rol crucial en la vida cotidiana, ya que participan en el procesamiento de la información. Según Banyard (1995, citado en Fuenmayor y Villasmil, 2008) estos se definen como estructuras o mecanismos mentales claves en el funcionamiento procesual; la percepción, el pensamiento, la memoria, el lenguaje y la atención son claves para el desempeño en cualquier actividad cotidiana, activándose cuando se realizan acciones de observación, lectura y escucha.

De acuerdo con Castillo y Paternina (2006), la atención, como proceso cognitivo, tuvo su primera aproximación teórica a finales del siglo XIX, gracias a W. James, quien logró esbozar una definición e incluso otorgarle una taxonomía, enfatizando su rol selectivo y relacional con la conciencia y las metas de los sujetos. Posteriormente, Luria en el año 1973 (citado en Manga y Ramos, 2011) la define como un proceso selectivo de toda la información que el ser humano necesita para la consolidación programática legible y su control adecuado en el curso de este mismo. En este sentido, Zanín et al. (2004) indican que puede considerarse una atención de tipo voluntaria y su contraparte involuntaria, manteniendo la vigilancia sobre un curso preciso y organizado, delegándole incluso indicadores fisiológicos. Esta situación ha sido reconceptualizada en favor de la complejidad y flexibilidad, en íntima dependencia con las exigencias de las tareas, pues es el factor responsable de extraer los elementos esenciales para el curso organizado y preciso de la actividad mental.

Aportes posteriores (Portellano Pérez y García Alba, 2005), sostienen que la atención es un mecanismo que permite la focalización selectiva de información o estímulos, filtrando o inhibiendo lo indeseado, para un posterior procesamiento. En este sentido, Rebollo y Montiel (2006) describen la atención como función independiente, de orden superior en relación con las funciones ejecutivas. 
http://doi.org/10.15359/ree.26-1.7

http://www.una.ac.cr/educare

educare@una.ac.cr

Monteoliva et al. (2014) hacen referencia a la complejidad conceptual, anatómica y neurofuncional, denotan que la atención no puede ser fácilmente reducida a una definición concreta, sino que debe ser valorada como una síntesis de variados sucesos cerebrales complejos, cuyos elementos forman parte del manejo procesal de información, que contribuye en la selección y predominio procedimental. En este sentido, se puede indicar que estos autores, consideran la atención como un mecanismo vertical de control, cuya función principal es dirigir recursos perceptivos a ciertos estímulos, activando o inhibiendo la elaboración y organización de la información, al ejercer significación participativa en el proceso de enseñanza y aprendizaje del estudiantado, pues pone en marcha diferentes modalidades de atención, inhibiendo y seleccionando información para su procesamiento casi en forma simultánea. La atención yace como mecanismo central en el control de los sistemas de procesamiento, no solo arraigada a nivel genético, sino también a factores psicosociales y familiares (Lagos et al., 2011).

Por otra parte, los procesos cognitivos, si bien pueden producirse de forma automática por su raíz biológica, deben de intervenirse en forma consciente por parte del sujeto, en una dominancia en favor del control e intencionalidad de estos mismos Viramonte (2000, citado por Fuenmayor y Villasmil, 2008). Para González et al. (2001) lo anteriormente descrito corresponde a una autorregulación voluntaria, que permite ejercer controles conscientes e intencionales que modulan la reactividad, la flexibilidad e intencionalidad atencional en la niñez, frente a diversas situaciones que sucedan en la vida, entre ellas, las instancias de aprendizaje, que si bien están previamente programadas, el progreso interno e intencional de regulación ante lo que selecciona, solo se promueve en dominancia de una manera individual.

La atención, además, ejerce una influencia significativa sobre la percepción, la memoria, el aprendizaje y en el control de la conducta, pues en ella, se domina el ingreso seleccionado de información en función de un procesamiento paralelo (Zanín et al., 2004). Este aspecto es complementado por Rivas Navarro (2008), quien afirma que, desde la infancia, los seres humanos deben seleccionar aspectos del contexto para percibirlo conscientemente, marcha que implica la fijación de dicha habilidad. Esta condición es, durante el desarrollo, limitada en términos de la cantidad de contenido, por la temporalidad en la mantención de la habilidad y su dirección hacia lo específico, pero aun así debe controlarse y regularse, como una manera de dominio sobre vivencia de experiencias de aprendizaje, provocándose entonces la meta-atención.

\section{Metacognición y autorregulación atencional al servicio del aprendizaje}

Para Glaser (1994, citado por Osses Bustingorry y Jaramillo Mora, 2008) el proceso metacognitivo configura como uno de los campos investigativos más influyentes y relevantes en la conformación y el desarrollo de principios de aprendizaje y de la instrucción. Para Jaramillo y Osses (2012) la metacognición es una habilidad auto modelada, cuyo protagonismo, 
http://doi.org/10.15359/ree.26-1.7

construcción, dirección y administración radica en el mismo estudiantado, ente independiente y responsable de su propio aprendizaje. Por este motivo guarda relación con la inteligencia intrapersonal, entendida como conocimiento personal de las fortalezas y debilidades, deseos, miedos, frustraciones y el actuar en base al mismo conocimiento en formas que permitan adaptarse a las circunstancias (Goleman, 2016).

La relevancia de la metacognición en el área educativa puede desprenderse desde los planteamientos de Osses Bustingorry y Jaramillo Mora (2008), quien sostiene que sin metacognición el proceso de enseñanza y aprendizaje, adquiere un rol pasivo, inoperante e irreflexivo de los educandos, cuyas ejecuciones automáticas de instrucciones, sin juicio, serían la vía y modo de aprendizaje preponderante.

De acuerdo con Román y Díez (2000, citado en Osses Bustingorry y Jaramilo Mora, 2008), los procesos metacognitivos conducen a un aprendizaje significativo por parte del alumnado, que se gesta en la relación congruente de tareas, la práctica y la decisión que el sujeto tiene por aprender, contrayendo parte de su propio conocimiento, relacionando conceptos y otorgándole un sentido a partir de la estructura conceptual.

En vínculo con la metacognición, Soto (2002, citado por Jaramillo y Osses, 2012) añade dos constituyentes de base, el primero de ellos, referido a la competencia metacognitiva, que implica operaciones dirigidas personas, quehaceres y estrategias, y el segundo, al moldeo del procesamiento, componente que incluye la planificación, la supervisión y la evaluación de procedimientos que sobresalen del nivel cognitivo, cuya concepción creacional superpone lo reflexivo ante lo cognitivo. En complemento con lo anterior, para Organista Díaz (2005) tener conocimiento sobre qué y cómo operan los procesos cognitivos como la atención, entre otros, facilitaría un mejor uso de los recursos cognitivos, permitiendo establecer cursos de acción más apropiados y efectivos para cada situación de aprendizaje. Por lo que no se puede analizar la metacognición, sin estimar la atención en el proceso de autorregulación en pro del aprendizaje, pues marca un vínculo constantemente colaborativo, cuya responsabilidad se relaciona con los logros académicos y el desarrollo de habilidades.

\section{Desarrollo neuronal y su implicancia en los procesos metacognitivos}

En torno a la atención y su regulación, se ha evidenciado la relación contigua con los lóbulos frontales del cerebro y, en particular, con las formaciones terciarias, cuya madurez no se alcanza hasta la edad de cuatro a siete años. Este vínculo, según autores como Luria o Struss y Benson (citados en Manga y Ramos, 2011), está directamente relacionado con la funcionalidad planificadora, reguladora, intencional y programática de dichas zonas cerebrales. 
http://doi.org/10.15359/ree.26-1.7

http://www.una.ac.cr/educare

educare@una.ac.cr

Al respecto, Anderson (citado en Manga y Ramos, 2011) plantea cuatro dominios ejecutivos relacionados con el desarrollo cronológico, argumentando que el control atencional es el primero en integrarse, sin embargo, entre los 7 y 9 años se experimenta un periodo crítico en el desarrollo de la flexibilidad cognitiva, establecimiento de metas y el procesamiento de la atención, debido a que en los años posteriores se determinará una maduración que influirá en la conducta. En consecuencia, el tramo etario mencionado sería un indicador importante al momento de fomentar habilidades atencionales, considerando la plasticidad neurológica abalada, posibilitando reorganizaciones funcionales del cerebro en desarrollo.

En Chile, las evaluaciones en torno a la atención se realiza en niñez sobre los siete años, destinadas a identificar sintomatología del trastorno de déficit atencional con hiperactividad o sin esta, en correspondencia con la normativa explicitada en el decreto 170/09 y destacada en el decreto número 83, mencionado por el Ministerio de Educación (2015), en función a la diversificación de la enseñanza, cuya intención es pesquisar y otorgar todos los apoyos necesarios, contemplando un marco inclusivo, que posibilite eliminar barreras para el aprendizaje de los niños y las niñas que padecen este trastorno. Dentro del marco normativo, se destina atención médica y profesional, además de un apoyo docente cualificado para las necesidades del estudiantado rotulados con esta etiqueta. Desde esta perspectiva, la atención, entendida como un déficit que forma parte de un trastorno es tratada con medicamentos. Por otra parte, la atención entendida como una habilidad a desarrollar en todo el estudiantado no es una temática que haga parte de la propuesta curricular vigente, por lo menos no desde una habilitación de la atención autorregulada. Esta restricción remueve de la atención su rama reflexiva, limitando con ello la facultad introspectiva de la atención.

\section{Mindfulness: Relación entre estado meditativo y atención autorregulada}

De acuerdo con Ricard et al. (2015), la práctica meditatoria conforma una acción inmemorial inserta en gran parte de las religiones del globo. La meditación, como práctica, ha sido referencia de arquetipo y ejemplo, fomentando la quietud, la salud y bienestar general. Los autores encasillan tres tipologías primordiales de meditación: la atención focalizada, la compasión y la atención o consciencia plena. Es una forma de involucrarse con la realidad presente, sin resistencia o interferencia, pudiendo ser considerada como una capacidad inherente a la conciencia humana.

"Mindfulness es la traducción al inglés de la palabra Sati (vocablo en lengua pali) que significa: consciencia, atención y recuerdo" (Mañas Mañas et al., 2014, p. 198). Bajo aquello, la consciencia es un estado de percepción y advertencia de lo que está ocurriendo dentro de la persona (pensamientos, emociones, sensaciones corporales, etc.) o fuera (olores, sonidos, personas, objetos, etc.). Es un estado de enfoque a algo determinado, que implica prestar atención a experiencias de concreción presente (Brown y Ryan, 2003) excluyendo pensamientos recurrentes, distracciones físicas e incluso emocionales.

6

Nicol Andrea González-Cortez y Nelly Lagos-San Martín

Los artículos de la Revista Electrónica Educare del Centro de Investigación y Docencia en Educación de la Universidad Nacional, Costa Rica, se comparten bajo términos de la Licencia Creative Commons: Reconocimiento, № Comercial, Sin Obra Derivada 3.0 Costa Rica. Las autorizaciones adicionales a las aquí delimitadas se pueden obtener en el correo: educare@una.cr 
http://doi.org/10.15359/ree.26-1.7

Para León (2008) es una forma contemplativa y perceptiva de las sensaciones experimentadas en el momento en que se producen, sin realizar valoraciones al respecto, sino viviendo la experiencia con apertura. Se trata de observar lo que ocurre en la vida, abarcando sensaciones, ideas y sentimientos, en un tiempo inmediato, sin realizar valor de juicio, ofreciendo interés, aceptación y curiosidad (Moñivas et al., 2012), aspecto que se destaca pues promueve a una apertura en sentido amplio, sin selección asociada a gustos o preferencias, que comúnmente ejerce el estudiantado ante nuevos estímulos implicados en el aprendizaje.

Otro acercamiento es el que plantea Rodríguez (2016), quien agrupa este conjunto de técnicas de atención y respiración, para ayudar a recuperar la presencia en el mundo y el vínculo con la existencia. Un camino bajo la meditación y observación de los procesos mentales, que abre los sentidos para percibir con mayor intensidad. En este sentido Moñivas et al., (2012) vinculan el mindfulness con procesos cognitivos, tales como la conciencia reflexiva y la metacognición, consideran que la práctica de ella conllevaría a descubrir que la actitud abstracta es la misma reflejada en la vida cotidiana en momentos de pasividad. De aquí su relación con la atención y la implicancia que tiene esta como proceso reflexivo destinado a ser enseñado para su dominio como una habilidad entrenada, no solo destinada a una función transversalmente pasiva, arraigada a la externalidad del estudiantado.

Bishop et al. (2004, citado por Mañas Mañas et al., 2014, pp. 200-201) plantean que existen "dos tipos de componentes implicados [en mindfulness]. El primero de ellos es el componente de autorregulación de la atención, cuya función es el mantenimiento y la redirección de la atención, además de la selección de estímulos concretos, [incluyendo] los procesos [de] atención sostenida, conmutación de la atención y [de] inhibición de la elaboración de pensamientos, sentimientos, sensaciones y emociones". Suponen focalizar la atención en lo que se hace o siente, facilitando, de esta manera, el devenir del organismo y de las propias conductas, relacionado con el segundo componente, el cual correspondería a la orientación hacia la experiencia que implica adoptar una relación particular hacia esta misma en el instante presente, caracterizada por una actitud de curiosidad, de apertura y de aceptación, situación que mejora los procesos de responsividad mental. Según este mismo autor, las habilidades mindfulness se encuentran relacionadas con las áreas de la atención sostenida, selectiva y focalizada, situación que confirma la relación directamente proporcional entre la práctica de mindfulness con los procesos atencionales y que produciría un cambio en el dominio de la habilidad, desde un foco interno, delegado en el estudiantado y no en situaciones externas que muchísimas veces no se pueden cambiar o manipular (León, 2008). Porter y Andrade (2013) rectifican, diciendo que la práctica del mindfulness podría incidir en la atención, y no tan solo en ella, sino también en la cognición, las emociones y el estrés, dado que provee un fortalecimiento en las funciones ejecutivas del cerebro, relacionadas con la autoconciencia y la autorregulación. Este planteamiento se dirige también al reconocimiento de sentimientos y sensaciones que fomentan su práctica, evitando la 
http://doi.org/10.15359/ree.26-1.7

http://www.una.ac.cr/educare

educare@una.ac.cr

extensión completa de ellas en la mente, como distractores, aspecto que provoca una detención seguida de una serenidad interior, disponible en todo momento y lugar, que se puede detener, por el tiempo necesario para elegir una respuesta sin influenciarse por automatismos.

Porter y Andrade (2013) también establecen que mindfulness permite mejores adaptaciones de la niñez a las demandas del aula, fomentando la permanencia de la atención como motivación hacia una tarea, así como la apertura flexible y consciente en el aprendizaje. Esta situación se registra bajo evidencia objetiva dentro de parámetros de carácter imageneológicos, pues cuando la meditación se enfoca o visualiza en la respiración, inhalación y exhalación, se produce un aumento de las señales de las estructuras cerebrales relacionadas con la atención: cortezas frontales, parietales, temporales y cingulada anterior, el hipocampo y el estriado que plantean Lazar et al. (2000, citado por Bonilla, 2008). La situán como práctica que amplía el funcionamiento de los hemisferios cerebrales, en sincronía con la actividad prefrontal.

El desarrollo de la atención plena puede ser primordial para el proceso de aprendizaje y para un desarrollo efectivo y emocional del estudiantado (León, 2008) ya que promueve la capacidad para realizar diversas tareas, sin frustrarse ante las equivocaciones, situación que provoca más posibilidades de aprendizaje, por la alta aceptación a las vivencias, dejando en un plano secundario los resultados de sus acciones, negativas o positivas, castigadas o premiadas. Por otro lado, Teasdale et al. (2015) afirman que mindfulness enseña a reconocer y elegir qué estrategia emplear y a cambiar los lineamientos automáticos, para actuar en forma consciente, lo que en otros términos refiere al desarrollo de habilidades metacognitivas.

Ruiz Lázaro et al. (2014) también hacen referencia a esta técnica desde un plano educativo, afirmando que la práctica de mindfulness otorga el beneficio de mejorar la atención de la niñez, rechazando automatismos y desarrollando un estilo de vida autoconsciente en el estudiantado, enfatizando además, que su práctica es de uso grato y sencillo en niñez, ya que puede abordarse con técnicas de juego y mantenerse siempre dentro de lo más práctico y directo, invitándoles a probar por sí y ver con curiosidad lo que les sucede en su propia experiencia, ayudando a generar un espacio distinto desde el cual se relacionan con sus sensaciones, emociones y pensamientos desde una apertura y aceptación (Porter y Andrade, 2013).

Sumando a lo respaldado anteriormente y desde las perspectivas de diferentes investigaciones, de acuerdo con Calvo et al, 2009; Delgado et al., 2010; Franco, 2009b; Kaspereen, 2012; López, 2007; Schonert-Reichl y Lawlor, 2010 (citados por Amutio-Kareaga et al., 2015) "se ha demostrado la utilidad de la aplicación de las técnicas de relajación y meditación/ mindfulness en el ámbito escolar, con la finalidad de aprender la relajación y de este modo activar los recursos internos de los estudiantes" (p. 433), relacionados con procesos regulativos en una activación consciente que promueve la dirección desde el estudiantado hacia su propio proceso atencional. Considerando lo anterior, se puede sostener que la atención es la antesala de todo aprendizaje, de modo que si esta manifestara alteraciones, la entrada y captación bajo 
diversas experiencias sostendría una amplitud limitada en beneficio del aprendizaje, mientras que si fuera adecuadamente estimulada, y por ende preparada, el enriquecimiento de las experiencias sería enormemente fructífero. Por ello entonces se ha diseñado una propuesta de intervención que se ha puesto a prueba de modo preliminar para determinar, si es efectiva en el desarrollo de la atención autorregulada en niñez de educación básica.

La hipótesis planteada en este estudio se refiere a que la atención sostenida, focalizada y dividida de estudiantes partícipes del plan de trabajo aumentaría luego de la implementación de estrategias de autorregulación de la atención mindfulness aplicadas al aula.

\section{Método}

El estudio se representa desde un enfoque tipo secuencial y probatorio, con planteamientos a investigar específicos y delimitados desde el inicio del estudio, con el propósito de determinar la incidencia que tienen las estrategias de autorregulación de la atención en el estudiantado de tercero básico, de la comuna de Pemuco. La recolección de datos se fundamenta en la medición y el análisis en procedimientos estadísticos, ahí el carácter cuantitativo que orienta a la elección de este enfoque.

Para realizar la investigación fue necesario manipular la variable independiente, para así detectar los efectos y establecer relaciones con la variable dependiente. Por lo mismo, el estudio de adscribió a un diseño pre-post, dos grupos, manteniendo uno de cuasi-control, donde se medirá en la variable dependiente en ambos grupos, en los mismos momentos en estudiantes de un curso determinado ( $3^{\circ}$ básico) que se escogieron antes de obtener la información.

\section{Participantes}

Los sujetos participantes de esta investigación no se asignaron aleatoriamente, puesto que estaban en grupos con anterioridad en dos cursos de un mismo nivel educativo (tercero básico) de un centro de educación básica, cuyos rangos etarios fluctuó entre 8 y 9 años de edad. Dicho tramo fue considerado en congruencia con el estado madurativo a nivel neurológico que se evidencia en dicho en estas edades, y porque además se ajusta a los test para medir los niveles atencionales de cada participante. Adicionalmente, señalar que ambos cursos pertenecían al único establecimiento municipal urbano de la comuna, razón por la cual se contaba con este número de estudiantes para el estudio. En total, los sujetos participantes sumaron 46 estudiantes, pertenecientes a dos cursos de 23 cada uno, con edades entre los 8 y 9 años.

\section{Técnicas de recogida de información}

Los instrumentos de medición de las variables medidas antes y después de la intervención fueron. 
http://doi.org/10.15359/ree.26-1.7

http://www.una.ac.cr/educare

educare@una.ac.cr

Test de CARAS: Instrumento creado por Thurstone y Yela (2012), permite medir atención selectiva o focalizada, evaluando la aptitud para percibir de forma rápida y correcta las semejanzas, diferencias y patrones parcialmente ordenados. Tiene una duración de tres minutos. La puntuación directa se basa en la totalidad de aciertos, con un máximo de 60 puntos.

Test CSAT: Instrumento creado por Servera Barceló y Llabrés Bordoy (2004) para medir atención sostenida mediante un método de vigilancia. El tiempo de respuesta estimado es de 7 minutos y medio, con una administración individual e informatizada. Está dirigida a niñez de 6 a 11 años. El número máximo de aciertos es 90 . La corrección de la evaluación es realizada por el mismo software, el cual arroja un informe que detalla los resultados obtenidos.

Subescala claves del test WISC-III, citado en Ramírez y Rosas (2007): En general, este test mide capacidades cognitivas. En esta investigación se utilizó una subescala denominada claves, puesto que se considera una prueba que mide la atención dividida/simultánea, en la cual el niño o la niña debe completar series, utilizando los símbolos faltantes durante dos minutos. Para calificar los resultados se utiliza la planilla de corrección que se equivale a puntuaciones normalizadas de acuerdo con el rango etario.

\section{Análisis de datos}

En primer lugar se realizó un análisis descriptivo y, dado que los datos indicaron la presencia de no-normalidad, se decidió realizar análisis no paramétricos para verificar si existen efectos estadísticamente significativos de los factores evaluados.

Las pruebas de contraste no-paramétrico utilizadas fueron las pruebas $\mathrm{U}$ de Mann Whitney, las cuales permitieron realizar comparaciones entre dos muestras independientes.

\section{Programa}

El programa de autorregulación de la atención basado en Mindfulness se sustenta en un plan de intervención de ocho semanas, las cuales se subdividen en sesiones semanales con una duración promedio de quince minutos. El personal docente se sitúa como monitor y guía de las dinámicas perceptivas y ejercicios para el procesamiento de información, que fomentan el desarrollo de habilidades atencionales autodireccionadas.

\section{Análisis de la información}

Se realizaron cálculos estadísticos para establecer diferencias significativas entre los resultados de la evaluación inicial y final de los grupos experimental y control. Los datos fueron analizados con el programa estadístico SPSS Stadistics versión 23. 
http://doi.org/10.15359/ree.26-1.7

Tabla 1: Diferencias de medias, significación estadística y magnitud diferencias del grupo experimental entre pretest y postest

\begin{tabular}{lccccccc}
\hline Variables & \multicolumn{2}{c}{ Pretest } & \multicolumn{2}{c}{ Postest } & \multicolumn{2}{c}{ Significación estadística y magnitud diferencias } \\
\hline & $M$ & $D E$ & $M$ & $D E$ & $t$ & $p$ & $D$ \\
\hline Test CARAS & 57,2 & 26,7 & 91,4 & 11,5 & $-5,13$ & $.000^{* *}$ & $-34,2$ \\
CSAT-Logro & 48,3 & 20,0 & 68,5 & 14,8 & $-3,54$ &, $001^{* *}$ & $-20,2$ \\
Test WISC & 22,2 & 4,5 & 29,4 & 5,3 & $-4,51$ &, $000^{* *}$ & $-7,2$ \\
\hline
\end{tabular}

Nota: Elaboración propia.

\section{Resultados}

Como puede observarse en la Tabla 1, para el grupo experimental (con intervención), en las tres pruebas existió un aumento en el puntaje promedio desde la aplicación inicial hasta el final, esto es: los puntajes promedio del test CARAS aumentaron en 34 unidades, los del test CSAT aumentaron en 20 unidades, y los del test WISC en 5 unidades, además su variabilidad ha disminuido en los tres casos.

Tabla 2: Diferencias de medias, significación estadística y magnitud diferencias del grupo control entre pretest y postest

\begin{tabular}{lccccccc}
\hline Variables & \multicolumn{2}{c}{ Pretest } & \multicolumn{2}{c}{ Postest } & \multicolumn{2}{c}{ Significación estadística y magnitud diferencias } \\
\hline & $M$ & $D E$ & $M$ & $D E$ & $t$ & $p$ & $D$ \\
\hline Test CARAS & 73,2 & 21,4 & 65,4 & 32,1 &, 809 &, 425 & 7,8 \\
CSAT-Logro & 47,7 & 18,3 & 53,4 & 21,0 &,- 881 &, 385 & $-5,7$ \\
Test WISC & 21,7 & 3,2 & 22,8 & 3,9 &,- 872 &, 390 & $-1,1$ \\
\hline
\end{tabular}

Nota: Elaboración propia.

En el grupo Control, solo el test CSAT obtuvo una mejora en sus puntajes. En particular: los puntajes del test CARAS disminuyeron en 8 puntos promedio, los del CSAT aumentaron solo 6 puntos, y los del test WISC solo un punto (véase tabla 2). 
http://doi.org/10.15359/ree.26-1.7

http://www.una.ac.cr/educare

educare@una.ac.cr

\section{Discusión}

De acuerdo con los datos, la aplicación del programa generó un aumento en las puntuaciones en la atención focalizada, dividida y sostenida respectivamente, observando resultados empíricamente positivos, de significación estadística, que demuestran efectividad en las tres tipologías atencionales examinadas, denotando valores sobresalientes en el aumento de atención focalizada, por sobre los resultados proporcionales de la atención sostenida y dividida correspondientemente. Los cambios favorables son corroborados también en el estudio realizado por Barragán Bech et al. (2007), quienes demuestran efectividad en la variación atencional dividida y sostenida, en una muestra de estudiantes, con edades disímiles.

Considerando el contexto nacional, en el que no se encuentran estudios previos ni vigentes que consideren un análisis atencional en función de la autorregulación en el rango etario, mucho menos considerando la base en mindfulness como recurso de apoyo, se estima que este programa es pionero en esta área $y$, habiendo mostrado un nivel de eficacia preliminar, se considera un aporte en el desarrollo de las habilidades de entrada y procesamiento de la información más relevante en el aprendizaje, vinculada transversal e implícitamente en el currículo.

Es relevante también señalar que el principal aporte de este estudio está en la comprensión de un aprendizaje basado en la atención como autodominio, pues esta habilidad en la realidad curricular, solo se relaciona bajo un automatismo asociado a estímulos externos, asignando significación externa a variables influyentes en el aprendizaje, sin conceder la envergadura que compromete el empoderamiento del estudiantado.

Así, el programa diseñado propone técnicas para que el propio estudiantado dirija, domine, direccione y modifique sus focos atencionales, desde un punto interno, lo que crea un recurso abundante y duradero, en independencia de componentes externalizados, como habitualmente se realiza. Una de las fortalezas del estudio es, a su vez, un impulso a la fragilidad del mismo, pues el sentido reciente e innovador, pese a promover la originalidad en pro de actualizaciones educativas que faciliten los procesos, también otorga un escaso efecto correlacional en una dirección contextual a nivel país. Dicha situación puede discutirse en distintos focos, desde una postura edificante, que fomenta proyectos originales y recientes, o desde un territorio adverso, por la limitada comparación con base en los efectos en realidades territoriales.

\section{Conclusiones}

Tomando en cuenta los resultados obtenidos en ambos cursos para las dos tandas, el progreso es claramente visible para el grupo experimental respecto del grupo control. Las metodologías de intervención basadas en mindfulness han sido exitosas en el curso intervenido dado que la autorregulación de la atención fue lograda de manera significativa, avalado por el impacto del programa en este alumnado. En este sentido, se observa que, en el grupo experimental, la

12 Nicol Andrea González-Cortez y Nelly Lagos-San Martín

Los artículos de la Revista Electrónica Educare del Centro de Investigación y Docencia en Educación de la Universidad Nacional, Costa Rica, se comparten bajo términos de la Licencia Creative Commons: Reconocimiento, No Comercial, Sin Obra Derivada 3.0 Costa Rica. Las autorizaciones adicionales a las aquí delimitadas se pueden obtener en el correo: educare@una.cr 
http://doi.org/10.15359/ree.26-1.7

variabilidad de las puntuaciones de los test es mayor que en el grupo control, lo que cambia completamente luego de la intervención mindfulness, razón por la cual puede añadirse que el alumnado intervenido homogeneizó sus resultados en favor de un avance positivo.

Como herramienta, la investigación ofrece a los grupos educadores un recurso práctico y efectivo, que en consecuencia impulsa y promueve la configuración de logros atencionales conscientes y autorregulados en estudiantes, situación que debe ampliarse en diferentes niveles de promoción.

Un admirable aporte se manifiesta en la sencillez, la economía, y precisión referida a la aplicabilidad del programa, que posibilita la realización de las dinámicas sin dificultar al profesorado. Dicha aplicabilidad se pretende profundizar en futuras investigaciones, que estén bajo los lineamientos Mindfulness en pro de la autorregulación atencional, considerando una ampliación muestraria para enriquecer los análisis y determinar generalizaciones según los resultados.

\section{Declaración de financiamiento}

Este estudio fue financiado por proyecto FONDECYT 11160040 de ANID, Chile.

\section{Referencias}

Amutio-Kareaga, A., Franco Justo, C., Gázquez Linares, J. J. y Mañas Mañas, I. (2015). Aprendizaje y práctica de la conciencia plena en estudiantes de bachillerato para potenciar la relajación y la autoeficacia en el rendimiento escolar. Universitas Psychologica, 14(2), 433-444. https:// doi.org/10.11144/Javeriana.upsy14-2.apcp

Barragán Bech, R., Lewis Harb, S. y Palacio Sañudo, J. E. (2007). Autopercepción de cambios en los déficit atencionales intermedios de estudiantes universitarios de Barranquilla sometidos al método de autocontrol de la atención (mindfulness). Salud Uninorte, 23(2), 184-192. http://rcientificas.uninorte.edu.co/index.php/salud/article/view/4032

Bonilla, E. (2008). Evidencias sobre el poder de la intención. Investigación Clínica, 49(4), 595-615. http://ve.scielo.org/pdf/ic/v49n4/art12.pdf

Brown, K. W. y Ryan, R. M. (2003). The benefits of being present: Mindfulness and its role in psychological well-being. Journal of Personality and Social Psychology, 84(4), 822-848. https://doi.org/10.1037/0022-3514.84.4.822

Fuenmayor, G. y Villasmil, Y. (2008). La percepción, la atención y la memoria como procesos cognitivos utilizados para la comprensión textual. Revista de Artes y Humanidades UNICA, 9(22), 187-202. http://www.redalyc.org/articulo.oa?id=170118859011 
http://doi.org/10.15359/ree.26-1.7

http://www.una.ac.cr/educare

educare@una.ac.cr

Goleman, D. (2016). El espíritu creativo. Ediciones B.

González, C., Carranza, J. A., Fuentes, L. J., Galián, M. D. y Estévez, A. F. (2001). Mecanismos atencionales y desarrollo de la autorregulación en la infancia. Anales de psicología, 17(2), 275-286. https://www.redalyc.org/articulo.oa?id=16717211

Jaramillo, S. y Osses, S. (2012). Validación de un instrumento sobre metacognición para estudiantes de segundo ciclo de educación general básica. Estudios pedagógicos, 38(2), 117-131. https://doi.org/10.4067/S0718-07052012000200008

Lagos, L. P., Silva, C., Rothhammer, P., Carrasco, X., Llop, E., Aboitiz, F. y Rothhammer, F. (2011). Riesgo de déficit atencional/hiperactividad en escolares Aymara, Rapa-Nuiy de Santiago de Chile. Posible contribución de polimorfismos genéticos del sistema dopaminérgico. Revista Médica de Chile, 139(5), 600-605. https://doi.org/10.4067/S0034-98872011000500006

León, B. (2008). Atención plena y rendimiento académico en estudiantes de enseñanza secundaria. European Journal of Education and Psychology, 1(3), 17-26. https://doi. org/10.30552/ejep.v1i3.11

Manga, D. y Ramos, F. (2011). El legado de Luria y la neuropsicología escolar. Psychology, Society \& Education, 3(1), 1-13. https://doi.org/10.25115/psye.v3i1.443

Mañas Mañas, I., Franco Justo, C., Gil Montoya, M. D. y Gil Montoya, C. (2014). Educación consciente: Mindfulness (Atención plena) en el ámbito educativo. Educadores conscientes formando a seres humanos conscientes. En R. L. Soriano Díaz y P. Cruz Zúñiga (Eds.), Alianza de civilizaciones políticas migratorias y educación (pp. 193-229). Aconcagua libros. https:// dialnet.unirioja.es/servlet/libro?codigo $=563553$

Ministerio de Educación. (2015). División de Educación Decreto número 83/2015. Aprueba criterios y orientaciones de adecuación curricular para estudiantes con necesidades educativas especiales de educación parvularia y educación básica. División de Educación General, Unidad de Currículum. https://especial.mineduc.cl/wp-content/uploads/ sites/31/2016/08/Decreto-83-2015.pdf

Monteoliva, J. M., Ison, M. S. y Pattini, A. E. (2014). Evaluación del desempeño atencional en niños: Eficacia, eficiencia y rendimiento. Interdisciplinaria, 31(2), 213-225. https://doi. org/10.16888/interd.2014.31.2.2

Moñivas, A., Gracia-Diex, G. y Gracía-de-Silva, R. (2012). Mindfulness (atención plena): Concepto y teoría. Portularia, 12(Extra), 83-89. https://doi.org/10.5218/prts.2012.0009

Organista Díaz, P. (2005). Conciencia y metacognición. Avances en Psicología Latinoamericana, 23, 77-89. https://marxismocritico.files.wordpress.com/2012/03/1108.pdf 
http://doi.org/10.15359/ree.26-1.7 http://www.una.ac.cr/educare educare@una.ac.cr

Osses Bustingorry, S. y Jaramillo Mora, S. (2008). Metacognición: Un camino para aprender a aprender. Estudios Pedagógicos, 34(1), 187-197. https://doi.org/10.4067/S0718$\underline{07052008000100011}$

Porter, B. y Andrade, M. (2013). La vida es ahora. Los beneficios del mindfulness en el día a día. El mercurio-Aguilar.

Portellano Pérez, J. A. y García Alba, J. (2005). Neuropsicología de la atención, las funciones ejecutivas y la memoria. Síntesis.

Ramírez, V. y Rosas, R. (2007). Estandarización del WISC-III en Chile: Descripción del Test Estructura Factorial y Consistencia Interna de las Escalas. Psykhe, 16(1), 91-109. https://doi. org/10.4067/S0718-22282007000100008

Rebollo, M. A. y Montiel, S. (2006). Atención y funciones ejecutivas. Revista Neurología, 42(2), 3-7. https://doi.org/10.33588/rn.42S02.2005786

Ricard, M., Lutz, A. y Davidson, R. (2015). En el cerebro del meditador. Las nuevas técnicas de neuroimagen arrojan luz sobre los cambios cerebrales que producen las prácticas contemplativas. Investigación y Ciencia, (460), 19-25. https://www.investigacionyciencia. es/revistas/investigacion-y-ciencia/neurociencia-de-la-meditacin-618/en-el-cerebro-delmeditador-12725

Rivas Navarro, M. (2008). Procesos cognitivos y aprendizajes significativos. Comunidad de Madrid.

Rodríguez, A. F. (2016). Cuaderno práctico de ejercicios de Mindfulness. Paidós.

Ruiz Lázaro, P. J., Rodríguez, T., Martínez Prádanos, A. y Núñez Marín, E. (2014). Mindfulness en pediatría: El proyecto "creciendo con atención y consciencia plena". Pediatría Atención Primaria, 16(62), 169-179. http://scielo.isciii.es/scielo.php?script=sci arttext\&pid $=\mathrm{S} 1139-76322014000300011$

Servera Barceló, M. y Llabrés Bordoy, J. (2004). CSAT-R.: Tarea de atención sostenida en la infancia. TEA Ediciones.

Teasdale, J., Williams, M. y Segal, Z. (2015). El camino del mindfulness. Paidós.

Thurstone, L. L. y Yela, M. (2012). CARAS-R. Test de percepción de diferencias - revisado (11.a ed.). Tea Ediciones.

Zanín, L., Gil, E. y de Bortoli, M. (2004). Atención y memoria: Su relación con la función tiroidea. Fundamentos en Humanidades, 5(10), 31-42. https://www.researchgate.net/ publication/26420116 Atencion y memoria su relacion con la funcion tiroidea 